\title{
基于固定化纳米金增强化学发光双酶传感器测定 葡萄糖
}

\author{
林洁华，张慧，张书圣 \\ 青岛科技大学化学与分子工程学院; 生态化工教育部重点实验室, 青岛 266042 \\ * 联系人, E-mail: shushzhang@126.com
}

收稿日期: 2008-09-12; 接受日期：2008-10-15

山东省自然科学基金 (批准号: Q2007B03)、青岛科技大学博士基金 (批准号: 0022141)和国家自然科学基金(批准号: 20775038)资助

\begin{abstract}
摘要研制了一种新型流动注射化学发光 $(C L)$ 双酶传感器, 用于葡萄糖的检 测. 该传感器将掺杂金纳米粒子(GNPs)的壳聚糖膜包覆在硅烷化试剂预处理的玻璃 微珠上, 用于吸附固定葡萄糖氧化酶(GOD) 和辣根过氧化物酶(HRP). 葡萄糖在 GOD 的催化下发生氧化反应生成 $\mathrm{H}_{2} \mathrm{O}_{2}$, 生成的 $\mathrm{H}_{2} \mathrm{O}_{2}$ 在 HRP 的催化作用下与鲁米诺 发生反应, 并产生化学发光信号. 实验表明, 壳聚糖中掺杂的GNPs 不仅能够有效的 吸附酶分子并保持其生物活性, 还对 Luminol- $\mathrm{H}_{2} \mathrm{O}_{2}-\mathrm{HRP}$ 化学发光体系具有增敏作
\end{abstract}

关键词

化学发光传感器

葡萄糖

金纳米粒子

辣根过氧化物酶

葡萄糖氧化酶

\section{1 引言}

实现葡萄糖的快速定量检测在生物化学、临床化 学以及食品分析等领域具有重要意义 ${ }^{[1]}$. 迄今为止, 已有许多有关葡萄糖检测方法的报道, 如电化学检 测 ${ }^{[1 \sim 6]}$ ，电致发光检测 ${ }^{[7]}$, 表面增强拉曼散射光谱检 测 [8], 光度法检测 [9]和化学发光检测 ${ }^{[10 \sim 15]}$ 等. 其中采 用葡萄糖氧化酶(GOD)和辣根过氧化物酶(HRP)研制 的双酶传感器, 由于具有选择性好, 灵敏度高等优点 而得到了广泛应用. 双酶反应体系的催化反应机理 如下:

$$
\begin{gathered}
\beta \text {-D-glucose }+\mathrm{O}_{2}+\mathrm{H}_{2} \mathrm{O} \stackrel{\text { GOD }}{\longrightarrow} \\
\text { gluconolacton }+\mathrm{H}_{2} \mathrm{O}_{2}
\end{gathered}
$$

$$
\underset{\text { 3-aminophthalic acid }+\mathrm{h} v}{\mathrm{H}_{2} \mathrm{O}_{2}+\text { luminol }} \stackrel{\mathrm{HRP}}{\longrightarrow}
$$

化学发光分析技术具有仪器设备简单，检测限 低和线性范围宽等优点. 近年来, 化学发光酶传感器 以其高灵敏度，高通量分析，易于操作和微型化等优 点引起了人们的广泛关注 ${ }^{[16 \sim 18]}$. 在诸多化学发光双 酶测定葡萄糖的研究报道中, 以鲁米诺化学发光体 系应用最为广泛. 例如: Economou $\mathrm{A}$ 等结合流动注射 /顺序注射分析技术，建立了一种快速测定葡萄糖的 方法，对葡萄糖检测的线性范围为 $0.01 \sim 1$ $\mathrm{mmol} / \mathrm{L}^{[10]}$. 一些催化剂如金属离子、金属贅合物、纳 米粒子和酶等都能有效的增强该体系的化学发光强 度 ${ }^{[19]}$. 近年来, 以金纳米粒子作为催化剂增强鲁米诺 
化学发光的研究相继有文献报道 $\underline{[20 \sim 25]}$. HRP作为 化学发光检测中应用最为普遍的酶之一, 通过催 化 $\mathrm{H}_{2} \mathrm{O}_{2}$ 氧化 Luminol 从而提高化学发光反应的效 率. 苯酚衍生物、二氧化碳、苂光素、苯基硼酸等化 合物均可作为该体系的发光增强剂 ${ }^{[26]}$, 可增强 Luminol- $\mathrm{H}_{2} \mathrm{O}_{2}-\mathrm{HRP}$ 体系的发光效率. Miró等报道了以 $\mathrm{Co}(\mathrm{II})$ 作为 Luminol- $\mathrm{H}_{2} \mathrm{O}_{2}$ - HRP化学发光体系的增强剂, 建立了一种双酶传感器测定葡萄糖的方法 ${ }^{[11]}$. 目前, 以固定化金纳米粒子 (GNPs) 作为增敏剂增强 Luminol- $\mathrm{H}_{2} \mathrm{O}_{2}$-HRP化学发光体系的研究还未见文献 报道.

章竹君等用蛋壳膜作为GOD和HRP的固定载体, 研制了一种新型的双酶传感器，对葡萄糖检测的线 性范围为 $0.001 \sim 0.1 \mathrm{mmol} / \mathrm{L}^{[15]}$. 壳聚糖作为一种性能 优良的天然聚合物，具有无毒、附着力强、生物兼容 性好以及对环境友好等优点 ${ }^{[27]}$, 可用作酶的固定化 载体. GNPs-壳聚糖复合膜能有效地维持生物分子的 活性，被视为一种新型的生物分子固定化载体 ${ }^{[28-30]}$. 本工作采用GNPs-壳聚糖膜固定HRP和GOD，构建了 一种双酶传感器, 采用流动注射化学发光分析测定 葡萄糖。首次提出以固定化GNPs作为增强剂，建立 了Luminol- $\mathrm{H}_{2} \mathrm{O}_{2}$-HRP-GNPs增强化学发光新体系, 并 对其增强机理进行了探讨.

\section{2 实验部分}

\section{1 试剂及标准溶液}

壳聚糖(MW $1.9 \sim 3.1 \times 10^{5}$; 脱乙酰度 $85 \% \sim 90 \%$, Aldrich), Luminol(ABCR GmbH \& Co. KG, 德国), $\mathrm{H}_{2} \mathrm{O}_{2}(\mathrm{AR}$ ，上海化学试剂厂), $\beta$-D-葡萄糖(AR，沈阳 试剂公司), $\gamma$-环氧丙氧基丙基三甲氧基硅烷(GPS，南 京化学试剂厂)，葡萄糖氧化酶(GOD，146 U/mg， Sigma), 辣根过氧化物酶(HRP, $300 \mathrm{U} / \mathrm{mg}$, Sigma), 玻璃微珠 $(<200 \mu \mathrm{m}$, 河北永清玻璃制品有限公 司). 其他试剂均为分析纯，使用时未经进一步提纯. $0.01 \mathrm{~mol} / \mathrm{L}$ Luminol 储备液: 称取 $0.1772 \mathrm{~g}$ 固体 Luminol 溶于 $0.1 \mathrm{~mol} / \mathrm{L} \mathrm{NaOH}$ 溶液中, 摇匀, 避光保 存, 使用时用 $0.1 \mathrm{~mol} / \mathrm{L} \mathrm{pH} 9.2$ 的 Tris- $\mathrm{HCl}$ 缓冲溶液 稀释至所需浓度. $2 \%$ 壳聚糖溶液 $(\mathrm{wt} \%)$ : 称取 $1 \mathrm{~g}$ 壳聚
糖固体粉末，溶于 $50 \mathrm{~mL} 1 \%$ 的醋酸溶液中，摇匀备 用. $1.0 \mathrm{mmol} / \mathrm{L} \mathrm{H}_{2} \mathrm{O}_{2}$ 溶液: 移取 $10.2 \mu \mathrm{L} 30 \% \mathrm{H}_{2} \mathrm{O}_{2}$ 于 $100 \mathrm{~mL}$ 蒸馏水中, 摇匀备用. 实验室用水为二次蒸 馏水.

\section{2 仪器}

IFFM-E 型流动注射化学发光分析仪(西安瑞迈 分析仪器有限公司), $0.1 \mathrm{~mol} / \mathrm{L} \mathrm{pH} 6.8$ 磷酸盐缓冲溶 液(PBS)作为葡萄糖的载流. JEM $1200 \mathrm{EX}$ 型透射电子 显微镜(JEOL, 日本). Cary 50 型紫外分光光度计 (Varian Pty Ltd，澳大利亚). 化学发光光谱在 F-4500 荧光分光光度计 (Hitachi, 日本)上测得, 检测时关闭 激发光源.

\subsection{GNPs-壳聚糖复合膜的合成}

采用柠檬酸钠还原法制得粒径分别为 25,40 及 $60 \mathrm{~nm}$ 的金溶胶 ${ }^{[30]}$, 通过TEM表征，得到的三种GNPs 的平均粒径分别为 $(25 \pm 2.5) \mathrm{nm},(40 \pm 3.0) \mathrm{nm}$ 及 $(60 \pm$ 5.0) $\mathrm{nm}$. 然后将金溶胶分别与 $10 \mathrm{~mL} 2 \%$ 的羧基化壳 聚糖溶液混合均匀。

用新配制的混酸 $\left(\mathrm{HNO}_{3}: \mathrm{HCl}=1: 3\right)$ 清洗玻璃微珠, 用二次蒸馏水彻底冲洗干净后晾干. 对玻璃微珠表 面进行硅烷化预处理后 ${ }^{[27]}$, 在其表面包覆一层GNPs壳聚糖膜，于 $40^{\circ} \mathrm{C}$ 下烘干 $2 \mathrm{~h}$.

\section{4 化学发光双酶葡萄糖传感器的研制}

将 GNPs-壳聚糖修饰的玻璃微珠分别置于 $1 \mathrm{~mL}$ $4 \times 10^{-4} \mathrm{~g} / \mathrm{mL}$ HRP 溶液和 $1 \mathrm{~mL} 5 \times 10^{-4} \mathrm{~g} / \mathrm{mL}$ GOD 溶液 中吸附酶分子 $24 \mathrm{~h}$, 并于 $4{ }^{\circ} \mathrm{C}$ 冰箱保存. 将固定了 $\mathrm{GOD}$ (或 HRP)的玻璃微珠填充到 $12 \mathrm{~cm}$ 长的透明塑料 管中(3.0 mm i.d.)制成 GOD(HRP)传感器. 如图 1 所 示, 在进行流动注射化学发光检测时, 当匍萄糖标准 溶液恰好充满 GOD 传感器时, 立即将管路的 $b$ 和 $d$ 两端连接起来形成回路. 当 $\mathrm{GOD}$ 与葡萄糖样品反应 完全后，断开 $\mathrm{b}$ 和 $\mathrm{d}$ 两端使管路恢复原状，此时反应 产生的 $\mathrm{H}_{2} \mathrm{O}_{2}$ 通过控制换向阀被载入到流通池内, 在 光电倍增管正上方的 HRP 传感器中与 Luminol 溶液 混合，在固定化 GNPs 的增敏作用下，由 IFFM-E 型 流动注射化学发光分析仪检测化学发光信号. 


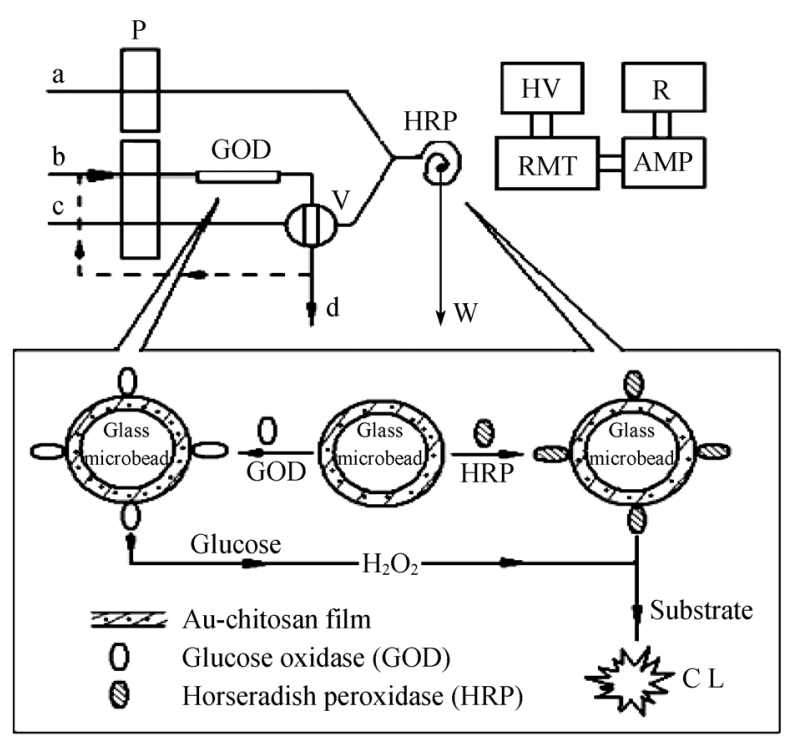

图 1 流动注射化学发光双酶传感器测定葡萄糖示意图

\section{3 结果与讨论}

\subsection{GNPs 对 Luminol- $\mathrm{H}_{2} \mathrm{O}_{2}-\mathrm{HRP}$ 化学发光体系 的增强作用}

研究了固定化GNPs对Luminol- $\mathrm{H}_{2} \mathrm{O}_{2}$-HRP化学发 光体系的影响. 结果表明, 壳聚糖中掺杂的GNPs显 著增强Luminol- $\mathrm{H}_{2} \mathrm{O}_{2}$-HRP体系的化学发光强度, 结 果如图 2 所示. 然而, 不同粒径的固定化GNPs对 Luminol- $\mathrm{H}_{2} \mathrm{O}_{2}-\mathrm{HRP}$ 化学发光体系的增强作用差别很 小(图 2 中未显示), 该结果与报道的溶液状态下, 不 同粒径金溶胶对 Luminol- $\mathrm{H}_{2} \mathrm{O}_{2}$ - $\mathrm{HRP}$ 化学发光体系增 强作用不同的结论不符 ${ }^{[25]}$.

Luminol- $\mathrm{H}_{2} \mathrm{O}_{2}$-HRP-固定化GNPs增强化学发光 体系的发光光谱如图 3 所示, 结果表明, Luminol$\mathrm{H}_{2} \mathrm{O}_{2}$-HRP-固定化GNPs增强化学发光体系的最大发 射波长为 $425 \mathrm{~nm}$, 与Luminol- $\mathrm{H}_{2} \mathrm{O}_{2}$ 化学发光体系的 最大发射波长相同. 表明该体系的发光体仍然是 3氨基邻苯二甲酸盐阴离子, 没有新的发光体生成. 化 学发光的增强作用可能是由于GNPs的催化效应, 加 速了体系的电子转移速率, 这与文献[25,31]报道的 机理相一致.

通过紫外光谱进一步研究了 Luminol- $\mathrm{H}_{2} \mathrm{O}_{2}-\mathrm{HRP}$ 固定化 GNPs 增强化学发光体系的发光机理, 如图 4 所示. 结果表明, 与金溶胶 (曲线(e))相比, 掺杂在壳

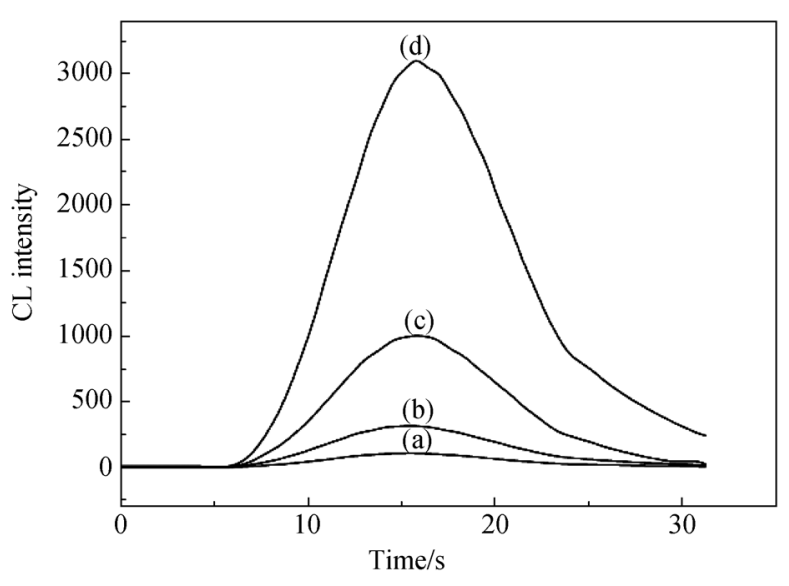

图 2 固定化 GNPs 对 Luminol- $\mathrm{H}_{2} \mathrm{O}_{2}$-HRP 化学发光体系 的增强作用

实验条件: $1.0 \mathrm{mmol} / \mathrm{L}$ Luminol 溶液; $0.1 \mathrm{~mol} / \mathrm{L}$ Tris- $\mathrm{HCl}$ 溶液 $(\mathrm{pH}$ 9.25); $1.0 \mathrm{mmol} / \mathrm{L} \mathrm{H}_{2} \mathrm{O}_{2}$ 溶液. (a) Luminol $+\mathrm{H}_{2} \mathrm{O}_{2}$; (b) luminol + $\mathrm{H}_{2} \mathrm{O}_{2}+$ GNPs; (c) luminol $+\mathrm{H}_{2} \mathrm{O}_{2}+\mathrm{HRP}$; (d) luminol $+\mathrm{H}_{2} \mathrm{O}_{2}+\mathrm{HRP}$ + GNPs

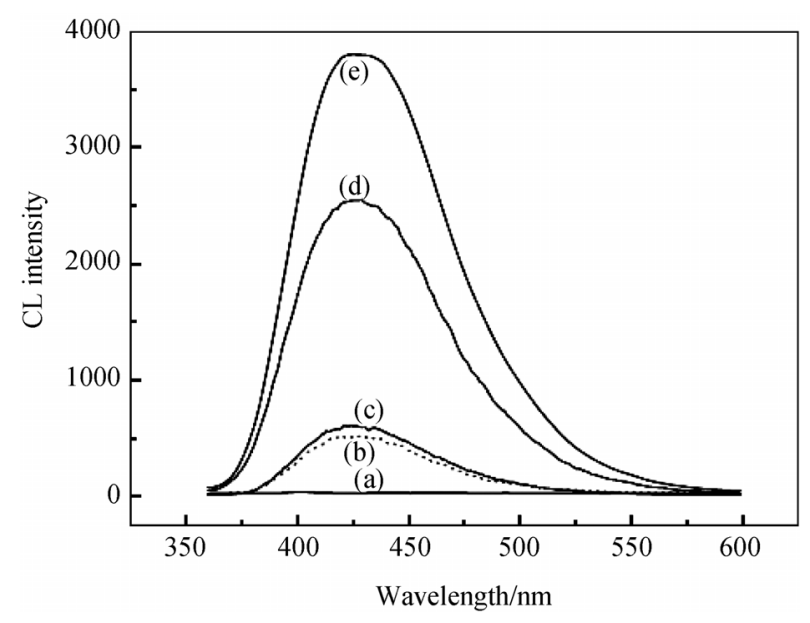

图 3 Luminol- $\mathrm{H}_{2} \mathrm{O}_{2}$-HRP-固定化 GNPs 增强化学发光体 系的化学发光光谱

实验条件: $1.0 \mathrm{mmol} / \mathrm{L}$ Luminol 溶液; $0.1 \mathrm{~mol} / \mathrm{L} \mathrm{pH} 9.25$ Tris- $\mathrm{HCl}$ 溶 液; $1.0 \mathrm{mmol} / \mathrm{L} \mathrm{H}_{2} \mathrm{O}_{2}$ 溶液. (a) Immobilized GNPs; (b) luminol + $\mathrm{H}_{2} \mathrm{O}_{2}$; (c) luminol $+\mathrm{H}_{2} \mathrm{O}_{2}+$ GNPs; (d) luminol $+\mathrm{H}_{2} \mathrm{O}_{2}+\mathrm{HRP}$; (e) luminol $+\mathrm{H}_{2} \mathrm{O}_{2}+\mathrm{HRP}+\mathrm{GNPs}$

聚糖中的GNPs的吸收波长 (曲线(f))没有出现红移, 表明固定在壳聚糖膜内的GNPs没有发生团聚现象. 固定化GNPs在化学发光反应前后, 紫外吸收波长没 有发生改变 (曲线(f), (g)), 该结果与文献报道的结果 相吻合 ${ }^{[25]}$, 进一步证明了GNPs的催化效应. 


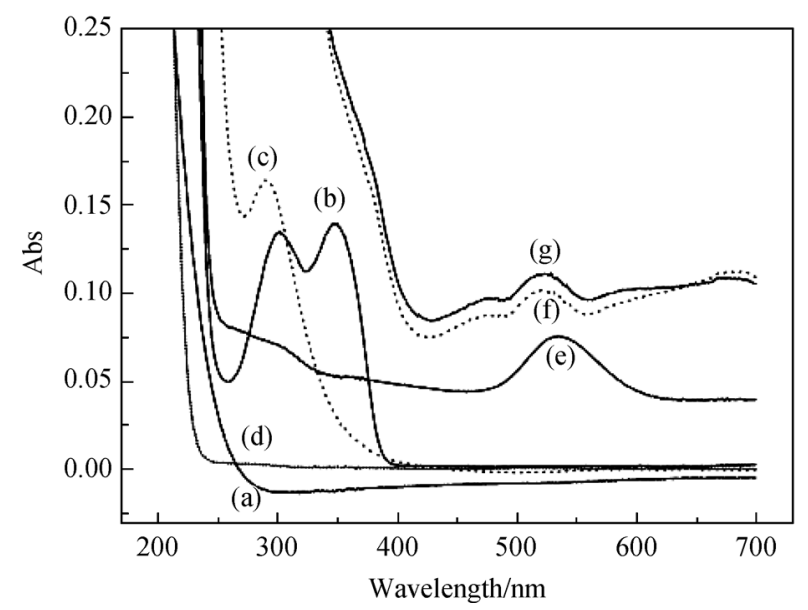

图 4 不同底物溶液的紫外光谱

(a) $\mathrm{H}_{2} \mathrm{O}_{2}$; (b) luminol; (c) $\mathrm{HAuCl}_{4}$; (d) 柠檬酸钠; (e) 溶液中的 GNPs; (f) 固定化的 GNPs; (g) 化学发光反应后固定化的 GNPs

综上所述，固定化GNPs对Luminol- $\mathrm{H}_{2} \mathrm{O}_{2}$-HRP化 学发光体系具有明显的增强作用, 并且, GNPs的粒径 大小对化学发光的增强作用影响不大. 由此推断, Luminol- $\mathrm{H}_{2} \mathrm{O}_{2}$ 体系化学发光的增强很可能是由掺杂 的GNPs和HRP的协同催化效应引起的. HRP能够催
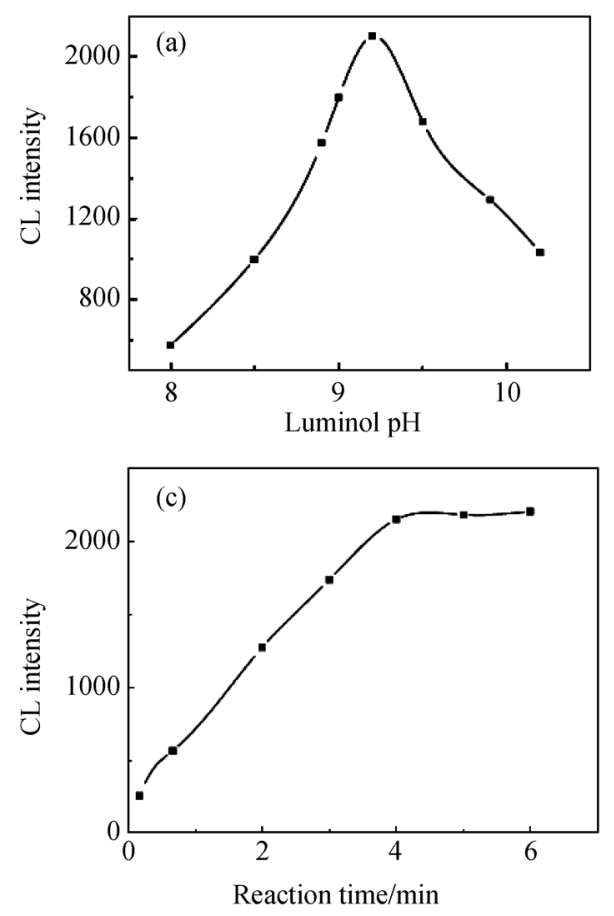

化 $\mathrm{H}_{2} \mathrm{O}_{2}$ 氧化Luminol的反应从而促进化学发光, 首先, $\mathrm{HRP}$ 与 $\mathrm{H}_{2} \mathrm{O}_{2}$ 反应形成氧化态HRP(HRP I), HRP I与 Luminol阴离子反应形成半还原态酶(HRP II)和激发 态Luminol ${ }^{[32]}$. 掺杂的GNPs 充当了反应的电子转移 媒介，加快了固定在其表面的HRP经HRP I和HRP II 转变为还原态(HRP)的速率, 从而起增强作用.

\section{2 化学发光检测条件的优化}

双酶传感器的性能主要取决于化学发光反应中 Luminol 溶液的浓度、缓冲溶液的 $\mathrm{pH}$ 值, 以及 GOD 催化氧化葡萄糖的反应条件. 优化检测条件的结果 如图 5 所示. 以 $0.1 \mathrm{~mol} / \mathrm{L} \mathrm{PBS}$ 缓冲液作为 $1.0 \mathrm{mmol} / \mathrm{L}$ 葡萄糖样品的载流, 在 GOD 传感器中反应 $4 \mathrm{~min}$, 从 而确定 Luminol 的反应 $\mathrm{pH}$ 和浓度. 图 5(a)表明, 当 $0.1 \mathrm{~mol} / \mathrm{L}$ Tris- $\mathrm{HCl}$ 缓冲溶液 $\mathrm{pH}$ 值为 9.2 时, 化学发 光信号最强. 图 5(b)表明, 当 Luminol 溶液浓度在 $0.05 \sim 1.0 \mathrm{mmol} / \mathrm{L}$ 之间时, 该体系的化学发光强度随 Luminol 溶液浓度的增加而增加, 当浓度达到 1.0 $\mathrm{mmol} / \mathrm{L}$ 时, 化学发光强度基本达到峰值, 然后随着 Luminol 溶液浓度的增加, 发光信号反而降低. 因此,
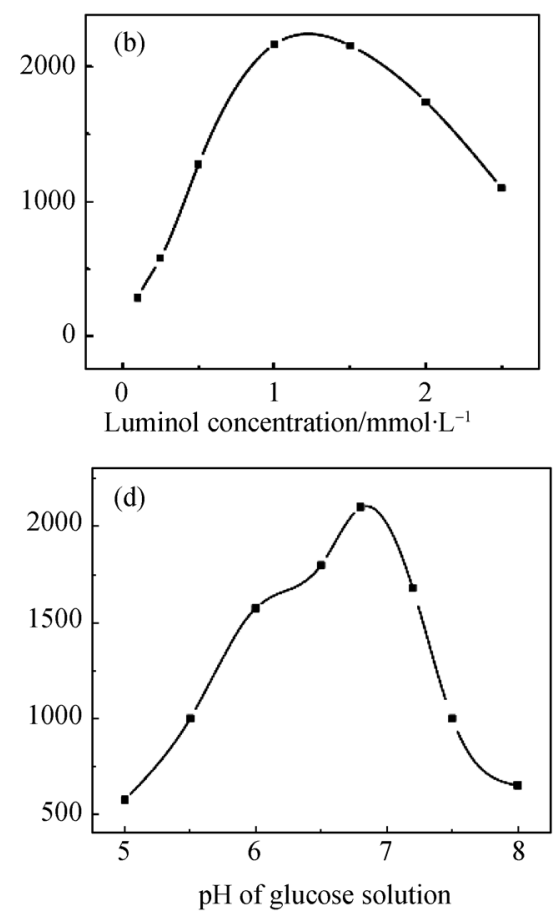

图 5 葡萄糖测定实验条件优化

(a) Luminol 溶液 pH 值; (b) luminol 溶液浓度; (c)葡萄糖的酶催化反应时间; (d)葡萄糖浓度. 实验条件: $1.0 \mathrm{mmol} / \mathrm{L}$ 葡萄糖溶液; $0.1 \mathrm{~mol} / \mathrm{L}$ PBS 缓冲液; $0.1 \mathrm{~mol} / \mathrm{L}$ Tris- $\mathrm{HCl}$ 缓冲液 
选择 Luminol 溶液的最佳 $\mathrm{pH}$ 值为 9.2, 最佳浓度 1.0 $\mathrm{mmol} / \mathrm{L}$.

在最佳实验条件下，试验了 GOD 催化氧化葡萄 糖反应时间的影响, 如图 5(c)所示. 结果表明, 葡萄糖 在 GOD 传感器中流通的时间越长, 两者的反应时间 越长, 产生的 $\mathrm{H}_{2} \mathrm{O}_{2}$ 的量越大. 但是, 反应时间太长会 使整个分析过程延长. 因此, 选择催化氧化反应时间 为 $4 \mathrm{~min}$. 试验了葡萄糖载流酸度的影响, 如图 5(d) 所示. 结果表明，当 PBS 缓冲溶液的 $\mathrm{pH}$ 值为 6.8 时， 体系的化学发光信号最强. 因此, 检测中选择 GOD 酶催化反应的最佳 $\mathrm{pH}$ 值为 6.8 .

\section{3 葡萄糖的检测}

在最佳实验条件下对葡萄糖标准溶液进行了测 定，结果如图 6 所示. 结果表明，对葡萄糖检测的线 性范围为 $0.01 \sim 6.0 \mathrm{mmol} / \mathrm{L}$, 线性方程为 $I=$ $42.87( \pm 27)+1566( \pm 11) C$ (其中, $I$ 为体系的化学发光 强度, $C$ 为葡萄糖浓度, $\mathrm{mmol} / \mathrm{L}$ ), 线性相关系数为 $0.9997(n=10)$, 检测限为 $5.0 \mu \mathrm{mol} / \mathrm{L}(3 \sigma)$. 与文献报道 的化学发光葡萄糖传感器 ${ }^{[10,13]}$ 相比, 本方法灵敏度 高，检测限低且线性范围宽.

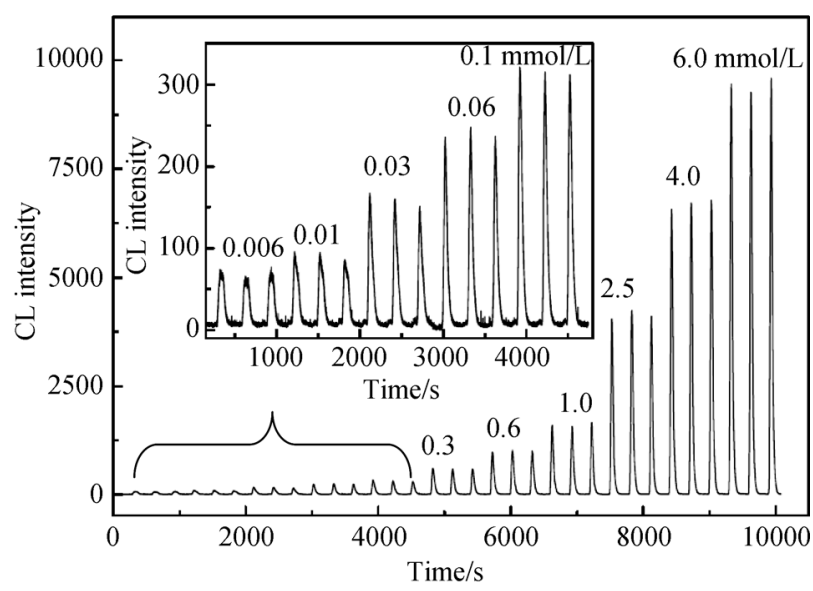

图 6 流动注射化学发光双酶传感器测定葡萄糖的工作 曲线

\section{4 干扰试验}

为检测血清中葡萄糖的含量, 研究了血清中共 存组分的干扰. 结果表明, 当葡萄糖溶液浓度为 0.5 $\mathrm{mmol} / \mathrm{L}$ 时, 1000 倍的 $\mathrm{Na}^{+}, \mathrm{K}^{+}, \mathrm{Ca}^{2+}, \mathrm{Cl}^{-}, \mathrm{NO}^{3-}, \mathrm{PO}_{4}{ }^{3-}$ 和 $\mathrm{SO}_{4}{ }^{2-} ; 100$ 倍的尿素和 50 倍的尿酸不干扰测定. 这 说明该生物传感器具有良好的选择性.

\section{5 血清样品测定}

对人血清中葡萄糖的含量进行了测定，见表 1 所 示. 结果表明，该方法和医院采用的临床分析方法所 得结果相吻合. 为进一步验证该方法的准确性, 采用 标准加入法测定了样品的回收率，见表 2. 结果表明, 回收率在 96\% 105\%之间，方法准确，可用于临床 样品的测定.

\section{表 1 血清样品中葡萄糖的测定}

\begin{tabular}{cccc}
\hline 样品 & $\begin{array}{c}\text { 医院临床 } \\
/ \mathrm{mmol} \cdot \mathrm{L}^{-1}\end{array}$ & $\begin{array}{c}\text { 本方法 } \\
/ \mathrm{mmol} \cdot \mathrm{L}^{-1}, n=5\end{array}$ & $\begin{array}{c}\text { 相对偏差 } \\
/ \%, n=5\end{array}$ \\
\hline Serum 1 & 0.29 & 0.23 & 2.62 \\
Serum 2 & 1.28 & 1.37 & 1.28 \\
Serum 3 & 5.60 & 5.28 & 0.87 \\
Serum 4 & 4.90 & 5.40 & 1.75 \\
\hline
\end{tabular}

表 2 样品中葡萄糖的回收率测定

\begin{tabular}{ccccc}
\hline 样品 & $\begin{array}{c}\text { 标液加入量 } \\
\mathrm{mmol} \cdot \mathrm{L}^{-1}\end{array}$ & $\begin{array}{c}\text { 测得值 } \\
/ \mathrm{mmol} \cdot \mathrm{L}^{-1}, n=5\end{array}$ & $\begin{array}{c}\text { 回收率 } \\
/ \%, n=5\end{array}$ & $\begin{array}{c}\text { 相对偏差 } \\
/ \%, n=5\end{array}$ \\
\hline 1 & 0.50 & 0.48 & 96.00 & 2.32 \\
2 & 1.50 & 1.57 & 104.67 & 1.86 \\
3 & 3.50 & 3.58 & 102.28 & 1.51 \\
4 & 4.50 & 4.38 & 97.33 & 2.70 \\
\hline
\end{tabular}

\section{6 传感器的稳定性}

传感器的稳定性主要取决于固定的 HRP 和 GOD 的稳定性. 对所研制的传感器的稳定性进行实验, 每次测完后将其置于 $\mathrm{pH} 7.0 \mathrm{PBS}$ 缓冲溶液中并于 4 ${ }^{\circ} \mathrm{C}$ 冰箱保存. 结果表明, 传感器保存 3 周后测得的发 光信号没有明显的降低，5周后测得信号大约降低到 初始值的 $85 \%$, 之后的两个月内信号基本保持不变. 另外, 为确定该传感器的操作稳定性, 对浓度为 1.0 $\mathrm{mmol} / \mathrm{L}$ 的葡萄糖标准溶液进行了 50 次连续测定，相 对标准偏差为 $1.9 \%$. 说明固定在 GNPs-壳聚糖膜上 的酶能够保持良好的生物活性，传感器具有较好的 稳定性. 


\section{4 结论}

本文构建了一种快速、高效的测定葡萄糖含量的 流动注射化学发光双酶传感器. 以 GNPs 掺杂的壳聚 糖膜作为 HRP 和 GOD 的固定化载体, 可以有效地固 定 HRP 和 GOD 酶分子, 而掺杂的 GNPs 对 Luminol$\mathrm{H}_{2} \mathrm{O}_{2}$-HRP 化学发光体系具有增敏作用. 固定化的 GNPs 充当了反应的电子转移媒介并加速了化学发光
反应中酶的催化作用. 由于固定化的 HRP 与 GNPs 的协同催化效应，该方法对葡萄糖的检测灵敏度高， 检测限低, 且线性范围宽. 所构建的生物传感器已成 功地用于血清样品中葡萄糖的测定. 固定化 GNPs 作 为 Luminol- $\mathrm{H}_{2} \mathrm{O}_{2}-\mathrm{HRP}$ 化学发光体系的一种新型增强 剂, 在酶分析和免疫分析等领域具有较好的应用 价值.

\section{参考文献}

1 De Benedetto G E, Palmisano F, Zambonin P G. One-step fabrication of a bienzyme glucose sensor based on glucose oxidase and peroxidase immobilized onto a poly(pyrrole) modified glassy carbon electrode. Biosens Bioelectron, 1996, 11: 1001-1008[DOI]

2 唐芳琼, 孟宪伟, 陈东, 再均国, 苟立, 郑昌琼. 纳米颗粒增强的葡萄糖生物传感器. 中国科学 B 辑: 化学, 2000, 30: $119-124$

3 Delvaux M, Walcarius A, Demoustier C S. Bienzyme HRP-GOx-modified gold nanoelectrodes for the sensitive amperometric detection of glucose at low overpotentials. Biosens Bioelectron, 2005, 20: 1587-1594[DOI]

4 Zhu L D, Yang R L, Zhai J L, Tian C Y. Bienzymatic glucose biosensor based on co-immobilization of peroxidase and glucose oxidase on a carbon nanotubes electrode. Biosens Bioelectron, 2007, 23: 528-535[DOI]

5 蔡称心, 陈静, 陆天虹. 碳纳米管修饰电极上葡萄糖氧化酶的直接电子转移. 中国科学 B 辑: 化学, 2003, 33: $511-518$

6 Ferri T, Maida S, Poscia A, Santucci R. A glucose biosensor based on electro-enzyme catalyzed oxidation of glucose using a HRP-GOD layered assembly. Electroanalysis, 2001, 13: 1198-1202 [DOI]

7 Marquette C A, Blum L J. Luminol electrochemiluminescenc-based fiber optical biosensors for flow injection analysis of glucose and lactate in natural samples. Anal Chim Acta, 1999, 381: 1-10 $\underline{\text { [DOI] }}$

8 Wu Z S, Zhou G Z, Jiang J H, Shen G L, Yu R Q. Gold colloid-bienzyme conjugates for glucose detection utilizing surface-enhanced Raman scattering. Talanta, 2006, 70: 533-539 [DOI]

9 Zhu M, Huang X M, Shen H X. Microbial enzymatic assay of glucose in serum. Anal Chim Acta, 1997, 349: 165-170[DOI]

10 Panoutsou P, Economou A. Rapid enzymatic chemiluminescent assay of glucose by means of a hybrid flow-injection/sequential-injection method. Talanta, 2005, 67: 603-609 [DOI]

11 Manera M, Miró M, Estela J M, Cerdà V. A multisyringe flow injection system with immobilized glucose oxidase based on homogeneous chemiluminescence detection. Anal Chim Acta, 2004, 508: 23-30[DOI]

12 Nicolau P, Manuel M, José M E, Víctor C. Automated enzymatic assays in a renewable fashion using the multisyringe flow injection scheme with soluble enzymes. Anal Chem, 2004, 76: 773-780[DOI]

13 Li B, Zhang Z, Jin Y. Plant tissue-based chemiluminescence flow biosensor for glycolic acid. Anal Chem, 2001, 73: 1203-1206 [DOI]

14 Zhang X R, Baeyenes W R G, Garcia C A M, Ouyang J. Recent developments in chemiluminescence sensors. Trends Anal Chem, 1999, 18: 384-391 [DOI]

15 Li B X, Lan D, Zhang Z J. Chemiluminescence flow-through biosensor for glucose with eggshell membrane as enzyme immobilization platform. Anal Biochem, 2008, 374: 64-70[DOI

16 Li B, Zhang Z. Chemiluminescence flow biosensor for determination of total D-amino acid in serum with immobilized reagents. Sensor Actuat B, 2000, 69: 70-74 $\underline{\text { DOD }}$

17 Aboul-Enein H Y, Stefan R I, van Staden J F, Zhang X R, Garcia C A M, Baeyens W R G. FA non-potentiometric sensing method for the determination of sulfite. Crit Review Anal Chem, 2000, 30: 271-289 [DOI]

18 Kiba N, Miwa T, Tachibana M, Tani K, Koizumi H. Chemiluminometric sensor for simultaneous determination of L-Glutamate and L-Lysine with immobilized oxidases in a flow injection system. Anal Chem, 2002, 74: 1269-1274[DOI]

19 Kricka L J, Voyta J C, Bronstein I. Chemiluminescent methods for detecting and quantitating enzyme activity. Meth Enzymol, 2000, 305: $370-390[\mathrm{DOI}]$ 
20 Niazov T, Pavlov V, Xiao Y, Gill R, Willner I. DNAzyme-functionalized Au nanoparticles for the amplified detection of DNA or telomerase activity. Nano Lett, 2004, 4: 1683-1687 $\underline{\text { DOI] }}$

21 Wang Z P, Hu J Q, Jin Y, Yao X, Li J H. In situ amplified chemiluminescent detection of DNA and immunoassay of IgG using special-shaped gold nanoparticles as label. Clin Chem, 2006, 10: 1958-1961[DOI]

22 Corgier B P, Li F, Blum L J, Marquette C A. On-chip chemiluminescent signal enhancement using nanostructured gold-modified carbon microarrays. Langmuir, 2007, 23 (16): 8619-8623 $\underline{\text { [DOI] }}$

23 Li Y X, Yang P, Wang P, Wang L. Development of a novel luminol chemiluminescent method catalyzed by gold nanoparticles for determination of estrogens. Anal Bioanal Chem, 2007, 387(2): 585-592[DOI]

24 Wang L, Yang P, Li Y X, Chen H Q, Li M G, Luo F B. A flow injection chemiluminescence method for the determination of fluoroquinolone derivative using the reaction of Luminol and hydrogen peroxide catalyzed by gold nanoparticles. Talanta, 2007, 72(3): $1066-1072$ [DOI]

25 Zhang Z F, Cui H, Lai C Z, Liu L J. Gold nanoparticle-catalyzed luminol chemiluminescence and its analytical applications. Anal Chem, 2005, 77: 3324-3329 [DOI]

26 Lin J H, Yan F, Ju H X. Noncompetitive enzyme immunoassay for carcinoembryonic antigen by flow injection chemiluminescence. Clin Chim Acta, 2004, 341: 109-115[DOD]

27 Lin J H, Yan F, Hu X Y, Ju H X. Chemiluminescent immunosensor for CA19-9 based on antigen immobilization on cross-linked chitosan membrane. J Immun Meth, 2004, 291: 165-174ㅁD]

28 Hao C, Ding L, Zhang X J, Ju H X. Biocompatible conductive architecture of carbon nanofiber-doped chitosan prepared with controllable electrodeposition for cytosensing. Anal Chem, 2007, 79: 4442-4447[DOI]

29 Huang H Z, Yang X R. Chitosan mediated assembly of gold nanoparticles multiplayer. Colloids Surf A: Physicochem Eng Aspects, 2003, 226: 77-86 $\underline{\text { [DOI] }}$

30 Lin J H, Qu W, Zhang S S. Disposable biosensor based on enzyme immobilized on Au-chitosan modified ITO electrode with flow injection amperometric analysis. Anal Biochem, 2007, 307: 288-293 [DOI]

31 Wallace W T; Whetten R L. Coadsorption of $\mathrm{CO}$ and $\mathrm{O}_{2}$ on selected gold clusters: Evidence for efficient room-temperature $\mathrm{CO}_{2}$ generation. J Am Chem Soc, 2002, 124: 7499-7505[DOI]

32 Dodeigne C, Thunus L, Lejeune R. Chemiluminescence as diagnostic tool. Talanta, 2000, 51: 415-439 [DOI] 